\title{
Low Attentional Control and the Development of Intrusive Memories Following a Laboratory Stressor
}

\author{
Johan Verwoerd • Peter J. de Jong • Ineke Wessel
}

Published online: 22 February 2008

(C) The Author(s) 2008

\begin{abstract}
This study tested whether low attentional control set people at risk for experiencing undesirable intrusions. Participants completed measures of attentional control and neuroticism and subsequently watched an emotional film fragment. In the four days following the presentation of the fragment, half of the participants $(n=17)$ were asked to keep a diary for the registration of intrusive memories. The other half of the participants $(n=16)$ only rated the number of intrusions retrospectively during the follow-up session. Low attentional control had independent predicting properties for the development of intrusive symptoms in the diary group. No such relationship was found in the no-diary controls, probably due to the relatively low frequency of intrusive symptoms that was elicited in this group.
\end{abstract}

Keywords Attentional control - Intrusive memories · Neuroticism $\cdot$ Intrusion diary

This research was supported by grant 452-03-329 of the Foundation for Behavioral and Educational Sciences of the Netherlands Organization for Scientific research (NWO).We thank Sippie Overwijk for her helpful comments on an earlier draft of this paper.

In this manuscript we use the term attentional control to refer to a general executive ability to hold or maintain the attentional spotlight on relevant information in working memory or the outside world (internally and externally focused attention). This could be accomplished by the inhibition of irrelevant information or the activation of relevant information.

J. Verwoerd $\cdot$ P. J. de Jong $\cdot$ I. Wessel

University of Groningen,

Groningen, The Netherlands

J. Verwoerd $(\triangle)$

Department of Clinical and Developmental Psychology,

University of Groningen,

Grote Kruisstraat 2-1, 9712 TS,

Groningen, The Netherlands

e-mail: j.r.l.verwoerd@rug.nl
One of the hallmark symptoms of posttraumatic stress disorder (PTSD) is the repeated occurrence of unwanted intrusive memories of the traumatic event. It appears that most people, after having experienced a traumatic situation, get rid of these overwhelming intrusive memories within one month (see Rothbaum et al. 1992), while a minority of them will stay highly vulnerable for cues triggering involuntary memories and will eventually develop PTSD. Considering this, vulnerability for persistent involuntary reliving of former traumatic events could imply a dysfunction in the mechanisms of memory control.

Germane to this, a series of recent cross-sectional studies using analogue samples provided evidence indicating that a relatively low level of executive control over the contents of working memory (i.e., working memory capacity $[\mathrm{WMC}]$ ) is associated with a relatively frequent occurrence of intrusive cognitions (Brewin \& Beaton 2002; Brewin \& Smart 2005; Klein \& Boals 2001). It has been proposed that individual differences in WMC are related to a domaingeneral capability to control attention, which is particularly important in situations involving proactive interference or conflict between competing response tendencies (Engle 2002). For example, in a study with normal undergraduate students, the frequency of experiencing intrusive memories and attempts to avoid such memories were relatively high in people who scored relatively low on a behavioral index of WMC (i.e., Operation Span; Klein \& Boals 2001). In line with this, it has been shown (Brewin \& Beaton 2002; Brewin \& Smart 2005) that individuals with a relatively high WMC are better able to suppress unwanted thoughts. Finally, using a self-report measure of distractibility (which might be taken as an index of deficient attentional control) it was found that people who described themselves as highly distractible also reported a relatively high frequency of intrusive memories (Verwoerd \& Wessel 2007). These 
earlier results are in line with the idea that deficient attentional control functions as a general vulnerability factor for development and maintenance of intrusive memories. However, because these studies were all crosssectional in nature it remains to be seen whether indeed low levels of attention or working memory control set people at risk for experiencing intrusive memories. To further explore this issue the present study was intended as a first step to test the predictive properties of (self-reported) attentional control on the development of experimentally induced intrusive memories using a prospective design.

A common laboratory method of investigating the development of intrusive memories is the use of a stressful film fragment. The stressful film paradigm creates an analogue situation in which response to trauma can be investigated in a controlled way (Holmes et al. 2004). A number of studies investigating information processing abnormalities have used this method to study the development of intrusive memories in the days after watching a highly stressful film (Brewin \& Saunders 2001; Davies \& Clark 1998; Holmes et al. 2004; Stuart et al. 2006). In most of these studies, participants were presented with a diary for the online registration of the frequency and characteristics of their intrusions rather than via an unexpected presentation of retrospective self-report questionnaires at a follow up session. There may be two consequences of using a diary for the registration of intrusive memories. On the one hand, using a diary may undermine the sensitivity of the present design to find a (negative) relationship between attentional control and the frequency of intrusive memories. That is, the frequency of reported diary intrusions may be influenced by the ability to keep the task goal of online diary registration in an active state. This may result in an artificially inflated number of diary intrusions in participants high in attentional control (potentially even reversing the theoretically expected negative correlation between attentional control and intrusive memories). On the other hand, the use of a diary may generally increase the activation level of the stressful memory. Yet, it may well be that the material used in analogue studies lacks sufficient emotional intensity to elicit intrusive memories very easily. If so, an increase in memory activation levels through the use of a diary may be a prerequisite for triggering a sufficient number of intrusive memories in order to reliably detect individual differences in the development of intrusive memories. Therefore, the present study included both a diary group and a no-diary control group to test the influence of using a diary on the number of reported intrusions as well as on the relationship between attentional control and intrusive memories.

The second aim of the present study was to investigate the role of neuroticism on intrusive memory development. It has been suggested that people high in the personality trait neuroticism may display distorted cognitive/information processing patterns which predispose them to show strong emotional reactivity and instability when confronted with threatening and stressful life events (Muris 2006). In line with this, earlier studies using clinical samples found a relationship between neuroticism and PTSD symptom severity (e.g., Morgan et al. 1995; Vassiliki \& Tarrier 2001; but see Engelhard et al. 2003 for a different interpretation). Furthermore, neuroticism has also been found to be strongly related with attentional control ( $r$ 's between -0.40 and -0.57 ; e.g., Derryberry \& Reed 2002; Muris 2006; Muris et al. 2004). Therefore, to get a better idea of the unique contribution of attentional control, the current study controlled for any mediating role that elevated levels of neuroticism might have on the relationship between low levels of attentional control and intrusive memories.

In sum, the main goal of the present study was to investigate the predictive value of deficient attentional control on the development of intrusive memories related to an earlier (experimentally elicited) aversive event. It was expected that relatively low levels of attentional control would predict relatively high levels of film-related intrusive memories in the four days after watching a stressful film. Additionally, it was expected that the diary method for the registration of intrusions would affect the predictive value of attentional control on self-reported intrusive and avoidance symptoms at follow-up. Finally, the role of neuroticism as a vulnerability factor and possible mediator was explored. It was expected that relationship between attentional control and intrusive memories would still exist after controlling for the shared variance with neuroticism.

\section{Method}

\section{Participants}

Thirty-three undergraduates participated in this study (18 men and 15 women). The mean age was 20.68 years $(\mathrm{SD}=2.89$, range 18-47). Participants (contacted by email or phone) were told that they would see a shocking film fragment with possible distressing effects. Following recruitment, they were randomly allocated into either a "diary group" or a "control group". Seventeen participants (eight men, nine women) were included in the diary group and sixteen participants were included in the control group (10 men, six women). All participants gave their written informed consent to take part. The University of Groningen Behavioral Research Ethical Committee granted ethics approval for this study.

\section{Stressful Film Fragment}

The experimental manipulation used in this study consisted of a 9-min fragment of "Irreversible" produced by Gaspar Noé. 
This film contains shocking and gruesome images of a brutal murder in a dark nightclub with very explicit and extreme violence. Subjects were not acquainted with the film before seeing the fragment. With respect to possible negative side effects of showing a film with a shocking content, participants were explicitly informed during recruitment and the experimental session prior to viewing the video that the film contained extreme violence and that they could leave the room and terminate the experiment at any point. The fragment was projected on a large $(150 \times 120 \mathrm{~cm})$ screen in a darkened room.

\section{Measures}

Attention and distress In order to measure the amount of attention paid to the film fragment, participants rated the percentage of time they had looked away from the screen $(0=$ not at all $-100=$ all of the time). They also reported on a $100 \mathrm{~mm}$ Visual Analog Scale (VAS; $0=$ not at all $-100=$ extremely) how much distress they experienced while viewing the fragment.

Attentional control In this study, we used a Dutch translation of the Attentional Control Scale (ACS, Derryberry \& Reed 2002; Verwoerd et al. 2006).The ACS is a 20-item self-report measure which measures attentional focusing (i.e., the ability to focus attention on one task: "When I need to concentrate and solve a problem, I have trouble focusing my attention") and attentional shifting (i.e., the ability to shift attention from one task to another and to engage in dual task behavior: "It is easy for me to alternate between two different tasks"). Scores on the ACS can range from 20 to 80 and the response format uses a four-point scale $(1=$ almost never; 2 = sometimes; 3 =often; 4 = always $)$, higher scores indicating good attentional control. The internal consistency of the ACS was found to be acceptable in the present sample (Cronbach's $\alpha=0.71$ ).

Neuroticism Neuroticism was measured with the Five Factor Personality Inventory (FFPI; Hendriks, Hofstee \& de Raad 1999) which is a self-report questionnaire for measuring the five super ordinate factors of temperament. The emotional stability scale of the FFPI consists of 20 items (e.g., "Is afraid to do something wrong") answered on a five-point scale $(1=$ does not at all apply to me, $5=$ does very much apply to me). For half of the items, the direction was reversed, so that higher scores would indicate higher neuroticism. The internal consistency of the emotional stability scale used in this study was high (Cronbach's $\alpha=0.93$ ).

Diary Participants in the diary group were asked to use an A5-format diary to record any intrusions of the films during the four days following the film (e.g., Brewin \& Saunders 2001; Davies \& Clark 1998; Holmes et al. 2004). They noted each intrusion's content ("Describe the content of your intrusion.") and rated the percentage of distress associated with experiencing the intrusion $(0=$ no distress $-100=$ high levels of distress). Elaborate verbal and written instructions were given about the nature of unwanted intrusions and how to keep the diary. As in re-experiencing symptoms in PTSD, intrusions were defined as "spontaneously occurring" (not intentional) memories of the film (see Holmes et al. 2004). Participants were asked to carry the diary with them and to record every intrusive memory related to the film fragment as soon as possible. The main dependent variable was the total number of intrusions reported in the diary. At follow up, participants had to fill in a diary compliance rating. They were asked to rate on a $100 \mathrm{~mm}$ VAS scale how well they had been able to keep track of their intrusions in the diary $(0=$ not at all $-100=$ very good $)$.

Impact of Event Scale A modified Dutch version of the Impact of Event Scale (IES; Horowitz et al. 1979; see van der Ploeg et al. 2004) instructed participants to respond to statements related to the film fragment they had been shown a few days earlier (e.g., images came up spontaneously). The modified scale consisted of 11 items answered on a fivepoint scale (range: $0-55$ ), with assigned item weights of 0,1 , 3,5 . The internal consistency of this scale was found to be high (Cronbach's $\alpha=0.89$ ).

\section{Procedure}

Participants were recruited from fellow students and friends of five research assistants who had to finish a second year research practical. In the week following the randomization, all participants filled in the ACS and were informed (by email or phone) about the time and day they were expected to participate in the experiment. Both the diary group and the control group watched the distressing fragment in group sessions on separate hours of the same day at the beginning of the week. Upon arrival at the test room, participants gave informed consent. After this, they were asked to fill in the neuroticism scale and were instructed that they could leave the room at any time. Before starting the distressing fragment, the experimenter gave instructions not to talk with each other and to avoid looking away as much as possible. Directly after viewing the film, participants rated the amount of attention they had paid to the film and how much distress they had experienced during the fragment. After this, participants in the diary group were instructed in the use of the 4-day diary while participants in the control group were only asked to come back after four days. The follow-up was conducted four days after the experimental 
manipulation. Participants in the diary group delivered their diaries and completed the diary compliance rating. All participants filled in the IES. Before leaving, the experimenter debriefed the participants about the purpose of the study.

\section{Statistical Analyses}

Hierarchical multiple regression analysis (HMR) was used to examine the main research questions of the predictive value of attentional control and the possible moderating role of using a diary on IES-scores at follow up. In the first step of this analysis, the variable group $(1=$ diary, $0=$ control) was included as a dummy variable, together with the attentional control scale. In the second step of the analysis, the moderating role of using a diary was tested by forcing the normalized product (interaction) of attentional control and group into the equation. In order to avoid further lowering of the participants/predictor ratio of the regression model, additional analyses used partial correlations to examine any mediating effects of neuroticism.

\section{Results}

One participant reported that she did not understand the questions on the neuroticism scale. Therefore, the analyses with neuroticism were conducted without this participant.

\section{General Results}

Mean, standard deviation and range of the main variables are shown in Table 1. The two groups (diary and control) did not differ on measures of attentional control or neuroticism and the percent of time looked away from the stressful fragment. Both groups also rated the distressing fragment as moderately emotional.
The effect of keeping a diary on the relationship between self-reported attentional control and IES-scores at follow-up

The HMR analysis with the IES as dependent variable, resulted in a significant $28 \%$ of explained variance in first step of the model, $F(2,30)=5.85, p<0.01$. Within this first step, the dummy variable group (diary, control), was a strong predictor of the variance in IES scores, $\beta=0.50, p<$ 0.01 . Participants who filled in a diary during the four days after viewing the distressing film fragment reported considerably more intrusions at follow-up $(M=11.47, \mathrm{SD}=10.91)$ than people in the control group $(M=4.06, \mathrm{SD}=4.41)$. However, the contribution of attentional control did not reach conventional levels of significance, $\beta=-0.24, t(31)=$ $-1.54, p=0.14$. The second step containing the interaction term between group and attentional control added a significant $9 \%$ to explained variance, $F$-change $(1,29)=$ 4.23, $p<0.05$. To further explore this interaction term, separate Pearson correlations between IES scores and attentional control were computed for the diary and the control group. It appeared that for participants who filled in a diary, self-reported attentional control strongly predicted follow up IES scores $[r(17)--.57, p<.05]$. In contrast, individual differences in attentional control for the participants in the control group did not have any predictive value for the later reported intrusive memories at follow-up $[r(16)=0.05, p=0.87]$.

Pearson correlations between neuroticism and IES-scores at follow-up

As shown in Table 2, the relationship between attentional control and neuroticism appeared to be moderately high $[r$ $(32)=-0.42, p<0.05]$. Furthermore, as expected, neuroticism was strongly associated with IES-scores at follow up $[r(32)=0.51, p<0.01]$. In general, there was a moderate to strong tendency for people with relatively high scores on

Table 1 General statistics (mean, standard deviation [SD] and range) of the main variables in the diary and control group

\begin{tabular}{|c|c|c|c|c|c|c|c|c|}
\hline & \multicolumn{3}{|c|}{ Diary $(N=17)$} & \multicolumn{5}{|c|}{ Control $(N=16)$} \\
\hline & Mean & $\mathrm{SD}$ & Range & Mean & SD & Range & $t$ & $P$ \\
\hline Attentional control & 53.06 & 5.18 & $43-64$ & 51.81 & 5.95 & $46-65$ & -.64 & n.s. \\
\hline Neuroticism & 44.41 & 11.04 & $26-61$ & 39.88 & 8.19 & $30-58$ & -.94 & n.s. \\
\hline Emotionality & 67.35 & 28.79 & $6-100$ & 55.81 & 23.97 & $11-80$ & -1.25 & n.s. \\
\hline Percent of time looked away & 6.59 & 9.28 & $0-34$ & 7.50 & 16.31 & $0-56$ & .20 & n.s. \\
\hline Follow-up (IES) & 11.47 & 10.94 & $0-38$ & 4.06 & 4.42 & $0-14$ & 3.21 & $<.01$ \\
\hline Diary intrusions & 2.59 & 1.84 & $0-7$ & & & & & \\
\hline Diary compliance & 64.24 & 21.84 & $10-96$ & & & & & \\
\hline
\end{tabular}

Emotionality emotionality ratings directly after viewing the film fragment, Percent of time looked away percent of time participants looked away from the film fragment, IES Impact of Event Scale 
Table 2 Pearson correlations between the main variables used in the present study

\begin{tabular}{lllll}
\hline & 1 & 2 & 3 & 5 \\
\hline 1. Attentional control & - & & & 4 \\
2. Neuroticism total score & $-0.42^{*}$ & - & & \\
3. Follow-up (IES) & -0.18 & $0.59^{* *}$ & - & - \\
4. Emotionality & -0.12 & $0.45^{* *}$ & $0.44^{* *}$ & 0.21 \\
5. Diary intrusions $(N=17)$ & $-0.58^{*}$ & 0.37 & $0.53^{*}$ & - \\
6. Diary compliance $(N=17)$ & -0.24 & -0.32 & 0.41 & 0.15 \\
\hline
\end{tabular}

IES Impact of Event Scale, Emotionality emotionality ratings directly after viewing the film fragment. ${ }^{*} p<0.05 ; * * P<0.01$

neuroticism to experience a relatively high frequency of intrusive memories.

The mediating role of neuroticism in explaining the relationships of attentional control with IES-scores and number of intrusive memories recorded in the diary

Since there was no significant association between attentional control and IES scores in the control group, all subsequent analyses are restricted to the diary group.

The results showed that, after controlling for neuroticism, there was still a trend towards significance for the correlation between attentional control and IES-scores at follow-up, $[\operatorname{pr}(13)=-.50, p=0.058]$. Additionally, the unique relationship between attentional control and the number of intrusive memories recorded in the diary was of considerable magnitude and in the same direction, although it failed to reach conventional levels of significance $[\operatorname{pr}(13)=-0.43$, $p=0.11]$. Taken together, these results suggest that weakened attentional control has independent predicting validity (over and above neuroticism) for the frequency of intrusive memories after an experimentally induced stressful situation. However, this predictive relationship was only evident in the group that used a diary as a method for the registration of intrusive memories.

\section{Discussion}

The major purpose of the current study was to explore the predisposing role of low attentional control and neuroticism on the development of intrusive memories in a prospective design. The main results can be summarized as follows. First, in line with previous studies, high levels of neuroticism were associated with a relatively high frequency of undesirable intrusive memories related to an experimentally induced aversive event. Second, for participants in the diary group, attentional control indeed had predictive value for the occurrence of subsequent intrusive and avoidance symptoms. Meanwhile, a similar relationship was absent for the no-diary control group.
The prognostic relationship between deficient attentional control and the frequency of undesirable intrusive memories in the diary group corroborate the results of prior studies relying on retrospective measures of intrusive memories. For example, earlier retrospective studies related vulnerability for experiencing intrusive memories to self-reported distractibility (Verwoerd \& Wessel 2007), to low working memory capacity (WMC) (Brewin \& Beaton 2002; Brewin \& Smart 2005; Klein \& Boals 2001), and to a reduced ability to resist or inhibit interference from working memory (Verwoerd et al. unpublished manuscript). Interestingly, the present findings give preliminary support our hypothesis that the predictive relationship between low attentional control and intrusive memory development would be largely independent of individuals' level of neuroticism. That is, attentional control uniquely predicted $25 \%$ of the variance in intrusive memories reported at the follow-up session. Meanwhile, consistent with the notion that neuroticism is a vulnerability factor for developing strong reactivity and instability when confronted with threatening/traumatic events (Muris 2006), neuroticism was shown to have predictive value for the development of intrusive memories. This pattern of results is in line with earlier analogue studies (Muris 2006; Muris et al. 2004) by showing unique contributions of attentional control and neuroticism in the context of intrusive memory development.

Some comments are in order to explain why the relationship between low attentional control and a high frequency of intrusive memories did only present itself in the diary group. The diary group reported almost three times as many IESintrusions at the follow-up session than the no-diary control group. This finding points to the possibility that keeping a diary for the registration of intrusive memories in analogue designs is a prerequisite to reliably evoke a meaningful number of intrusions in non-clinical individuals. That is, repeatedly thinking about the task goal of keeping a diary may increase the activation level of the stressful film memory and may subsequently trigger intrusive memories. Following this, for people in the diary group, good attentional control would be an indispensable ability to prevent undesirable intrusive memories from entering working memory. In contrast, for 
participants in the control group, the emotional fragment might not be sufficiently intense to develop a minimum number of intrusive memories. So, in this group, there was no need for executive attention to prevent the experience of undesirable intrusive memories.

On a more theoretical level, the question may rise how participants high in self-reported attentional control did prevent intrusive memories from entering working memory. Extending on earlier findings in the context of WMC (e.g., Klein \& Boals 2001; Kane \& Engle 2000), described as a domain-general capability to control attention, a recent study tested the idea that the ability to resist or suppress interference of irrelevant material from working memory might play a role in this. In this study, which used a retrospective design, individuals who reported a relatively high level of intrusive and avoidance symptoms on the IES showed a reduced ability to resist interference of first-list intrusions during second-list learning in an AB-AC-AB list-learning paradigm (Verwoerd et al. unpublished manuscript). Thus, a deficient ability to resist or suppress interference from working memory might be the underlying mechanism which sets people low in selfreported attentional control at risk for experiencing undesirable intrusive memories. Germane to this, the current findings of a relationship between low attentional or working memory control and intrusive memories might also contribute to further understanding the common finding of intelligence (IQ) as risk factor for the development and maintenance of PTSD symptoms (e.g., MacNally \& Shin 1995).

Some limitations of the present study need attention. First, the results of the present study relied primarily on the use self-report measures. Regarding the use of the attentional control scale, it might be possible that subjective reports about one's ability to control attention are not similar to more objective indices of attentional control. Therefore, further research is needed to test the predictive relationship of attentional control on the development of intrusive memories with more objective (experimental) measures. Second, it should be acknowledged that the size of the diary group was rather modest. So it seems important to investigate in future studies whether the main findings of the present study can be replicated in larger samples using extreme groups of high and low attentional control. Third, the selection of participants from fellow students of research assistants should be taken with caution. It is not clear to what extent these participants are representative of undergraduates in general, or the larger population. Future research should make use of more unbiased selection methods. Fourth, one further limitation/implication for future analogue diary studies might be that participants' strategies to keep track of experimentally elicited intrusive memories may not model the behavior of people in the aftermath of a real traumatic event in a correct way. That is, after a traumatic event, people try to avoid thinking about the trauma as much as possible instead of consciously keeping track of their intrusions. Meanwhile, strategies to avoid thinking about an aversive event may have a similar effect on intrusive remembering as the strategies used to keep the task goal of online diary registration in an active state as participants probably did in the present study.

In conclusion, the present findings are important in that they give preliminary evidence for a prospective relationship between attentional control and the development of intrusive memories after the experience of a stressful situation (e.g., film fragment). Furthermore, there was indication that the relationship between attentional control and intrusive memories was not mediated by elevated levels of neuroticism, preliminary supporting a unique predictive value. However, the most important findings of the present study were restricted to the participants who filled in a diary for the registration of intrusive memories. Possibly, the use of a diary supported the intrusiveness of the stressful film memory representation which in turn supported the detection of individual differences in the predictive value of attentional control. An important next step would be to replicate the present findings in larger samples using behavioral rather than self report measures of attentional control. Furthermore, the methodology of the present study might also be extended to clinical samples by measuring low attentional control and high neuroticism pre-trauma and investigating the subsequent incidence of PTSD.

Open Access This article is distributed under the terms of the Creative Commons Attribution Noncommercial License which permits any noncommercial use, distribution, and reproduction in any medium, provided the original author(s) and source are credited.

\section{References}

Brewin, C. R., \& Beaton, A. (2002). Thought suppression, intelligence, and working memory capacity. Behavior, Research \& Therapy, 40, 923-930.

Brewin, C. R., \& Saunders, J. (2001). The effect of dissociation at encoding on intrusive memories for a stressful film. British Journal of Medical Psychology, 74, 467-472.

Brewin, C. R., \& Smart, L. (2005). Working memory capacity and suppression of involuntary thoughts. Journal of Behavior Therapy and Experimental Psychiatry, 36, 61-68.

Davies, M., \& Clark, D. (1998). Predictors of analogue post-traumatic intrusive cognitions. Behavioral and Cognitive Psychotherapy, 26, 303-314.

Derryberry, D., \& Reed, M. A. (2002). Anxiety-related attentional biases and their regulation by attentional control. Journal of Abnormal Psychology, 111, 225-236.

Engelhard, I. M., Van den Hout, M. A., \& Kindt, M. (2003). The relationship between neuroticism, pre-traumatic stress, and posttraumatic stress: a prospective study. Personality and Individual Differences, 35, 381-388.

Engle, R. W. (2002). Working memory capacity as executive attention. Current Directions in Psychological Science, 11, 19-23. 
Hendriks, A. A. J., Hofstee, W. K. B., De Raad, B., \& Angleiter, A. (1999). The Five-Factor Personality Inventory (FFPI). Personality and Individual Differences, 27, 307-325.

Holmes, E. A., Brewin, C. R., \& Hennessey, R. G. (2004). Trauma films, information processing, and intrusive memory development. Journal of Experimental Psychology. General, 133, 3-22.

Horowitz, M. J., Wilner, N., \& Alvarez, W. (1979). Impact of Event Scale: A measure of subjective stress. Psychosomatic Medicine, 41, 9-18.

Kane, M. J., \& Engle, R. W. (2000). Working-memory capacity, proactive interference, and divided attention: Limits on long-term memory retrieval. Journal of Experimental Psychology. Learning, Memory, and Cognition, 26, 336-358.

Klein, K., \& Boals, A. (2001). The relationship of life event stress and working memory capacity. Applied Cognitive Psychology, 15, 565-579.

McNally, R. J., \& Shin, L. M. (1995). Association of intelligence with severity of posttraumatic stress disorder symptoms in Vietnam combat veterans. American Journal of Psychiatry, 152, 936-938.

Morgan, I. A., Matthews, G., \& Winton, M. (1995). Coping and personality as predictors of posttraumatic intrusions, numbing, avoidance and general distress: a study of victims of the Perth flood. Behavioral and Cognitive Psychotherapy, 23, 251-264.

Muris, P. (2006). Unique and interactive effects of neuroticism and effortful control on psychopathological symptoms in non- clinical adolescents. Personality and Individual Differences, 40, $1409-1419$.

Muris, P., de Jong, P. J., \& Engelen, S. (2004). Relationships between neuroticism, attentional control, and anxiety disorders in non-clinical children. Personality and Individual Differences, 37, 789-797.

Rothbaum, O. R., Foa, E. B., Riggs, D. S., Murdock, T., \& Walsh, W. (1992). A prospective examination of posttraumatic stress disorder in rape victims. Journal of Traumatic Stress, 3, 455-475.

Stuart, A. D. P., Holmes, E. A., \& Brewin, C. R. (2006). The influence of a visio-spatial grounding task on intrusive images of a traumatic film. Behavior Research and Therapy, 44, 611-619.

Van der Ploeg, E., Mooren, T. M., Kleber, R. J., Van der Velden, P. G., \& Brom, D. (2004). Construct validation of the Dutch version of the Impact of Event Scale. Psychological Assessment, $16,16-26$.

Vassiliki, H., \& Tarrier, N. (2001). Personality and peritraumatic dissociation in the prediction of PTSD in victims of road traffic accidents. Journal of Psychosomatic Research, 51, 687-692.

Verwoerd, J., \& Wessel, I. (2007). Distractibility and individual differences in the experience of intrusive memories. Personality and Individual Differences, 42, 325-334.

Verwoerd, J., de Jong, P. J., \& Wessel, I. (2006). ACS: Dutch translation of the Attentional Control Scale, originally developed by Derryberry and Reed (2002). 